\title{
Loop Current Excitations in Effectively Half-Filled Mott Insulators
}

\begin{abstract}
Hiroyasu Koizumi*
Institute of Materials Science, University of Tsukuba,Tsukuba, Ibaraki 305-8573, Japan

We show that spin vortex excitations in a two-dimensional half-filled antiferromagnetic insulator are accompanied by loop currents; they are the collective flow of electrons generated by a fictitious magnetic field, where the field arises from a phase factor that ensures the single-valuedness of wave functions for electron hopping motion. We study effectively halffilled states of a $U \gg t$ Hubbard model in which all holes added to the exactly half-filled antiferromagnetic insulator become self-trapped polarons. Using the perturbation theory, we demonstrate that loop currents arise when spin vortices are created and partially retrieve the itineracy of electrons in the upper Hubbard band. We also perform mean-field calculations to gain qualitative insights on the loop current state using parameters appropriate for cuprate superconductors. It is shown that a collection of loop currents creates a large current flow region or a "river" of current. A calculated ARPES profile exhibits a Fermi arc feature seen in cuprates when an extended loop current region is present; thus, it is suggested that such a current region may be the origin of the Fermi arc. The elastic neutron scattering cross section profiles are also calculated and a splitting of peaks that resembles experimental results is obtained.
\end{abstract}

KEYWORDS: Mott insulator, loop current, fictitious magnetic field, cuprate, Fermi arc

\section{Introduction}

Transition metal compounds often exhibit half-filled insulating states, or Mott insulator states $^{1)}$ due to strong electron correlation; and upon hole or electron doping, anomalous metallic states appear, where the doped holes or electrons are believed to be charge carriers. ${ }^{2)}$ In some compounds, however, due to strong electron-lattice (or hole-lattice) interaction, the doped holes or electrons become self-trapped small polarons; then, they are not effective charge carriers, especially at low temperatures. A peculiar point is that for some compounds, for example, cuprates or manganites, experimental and theoretical results suggest a strong interaction between the doped-carrier and the underlying lattice that leads to small polaron formation, yet also exhibit significant electric conductivity. ${ }^{3-13)}$

In the present work, a new insight into the above-mentioned peculiarity is presented; i.e., we present a current flow mechanism that is effective even if all the doped holes become selftrapped polarons. One might think that if all the doped carriers are immobile, electric current flow is impossible; however, we will show that if spin vortices are created, loop currents appear

*E-mail address: koizumi@ims.tsukuba.ac.jp 
around them, and a collection of loop currents give rise to a macroscopic current.

The novel loop current generation presented in this work arises from two effects brought about by spin vortex formation: one is the retrieval of the itineracy of electrons in the upper Hubbard band due to the destruction of an antiferromagnetic spin arrangement; and the other is an appearance of a fictitious magnetic field that works as an effective magnetic field for electron hopping motion. Here, the fictitious magnetic field arises because the presence of spin vortices causes a sign ambiguity in wave functions for electron hoping motion; then, a phase factor that ensures the single-valuedness of the wave function appears and gives rise to a vector potential for the fictitious magnetic field. Similar situations often occur in the context of the Berry phase problem. ${ }^{14-16)}$ Although the fictitious magnetic field is not the one that originates from electric current, its effect on electron motion is the same as the true one, and generates current.

The present work reverses a long-standing belief held for a half-filled antiferromagnetic state of the $U \gg t$ Hubbard model: it has been believed that at half-filling where the numbers of sites and electrons are equal, the effective Hamiltonian for the $U \gg t$ Hubbard model is the Heisenberg model; thus, possible low-energy excitations are those that include spin degrees of freedom only. ${ }^{17)}$ The present work shows that this belief is not correct; the mapping to the Heisenberg model ignores the spreading of wave functions through sites when the antiferromagnetic order is destroyed; if the spreading over the upper Hubbard band is taken into account, loop current generation occurs upon spin vortex excitations with excitation energies in the order of $t^{2} / U$.

In the present work, we consider a specific model: a Hubbard model for a two-dimensional square lattice where all holes doped into the exactly half-filled system are assumed to become self-trapped lattice polarons. In this situation, we can take into account the small polaron effect by simply eliminating hole-occupied sites from accessible sites for electron hopping; thus, the number of accessible sites and the number of electrons are equal even after the hole doping. We will be mainly concerned with this effectively half-filled situation in the present work.

This paper is organized as follows: in Section 2, we define new creation and annihilation operators, and rewrite the Hamiltonian using them. In this way, part of the hopping term can be included in the zeroth-order Hamiltonian; thereby, the spreading of wave functions through the upper Hubbard band can be taken into account. The fictitious magnetic field, a crucial ingredient for the current generation, also appears in this section. In Section 3, we rederive the well-known results that the ground state of the $U \gg t$ Hubbard model shows an antiferromagnetic correlation, ${ }^{17)}$ and the ground state of the same model with the number of electrons equal to the number of sites minus one is ferromagnetic. ${ }^{18)}$ The antiferromagnetic state here is different from that which arises from the Heisenberg model because part of 
the hopping term is included in the zeroth-order Hamiltonian. In Section 4, we explain the appearance of loop currents using the perturbation theory. Section 5 is a connection section to Section 6. A formula for the angle-resolved photoemission spectrum (ARPES) for the halffilled system is derived using a suitable mean-field solution that agrees with the one obtained by the degenerate perturbation theory; hence, the usefulness of this mean-field approach is demonstrated. In Section 6, the mean-field approximation derived in Section 5 is performed using parameters suitable for cuprates. The purpose of this section is to examine the features of the loop current states, qualitatively. It is demonstrated that the loop current is essentially proportional to the vector potential for the fictitious magnetic field. ARPES profiles are calculated, and a Fermi-arc-like feature is obtained. ${ }^{19)}$ The elastic neutron scattering cross section is calculated, and a splitting of the peak similar to the ones observed in experiments is obtained. ${ }^{20-23)}$ It is also demonstrated that a collection of loop currents may be able to generate an extended current region. Finally, in Section 7, we conclude the present work.

\section{New Creation and Annihilation Operators}

In this section, we introduce new creation and annihilation operators for electrons. The total Hamiltonian is a Hubbard model in the two-dimensional square lattice given by

$$
H=-\sum_{i, j, \sigma} t_{i j} c_{i \sigma}^{\dagger} c_{j \sigma}+U \sum_{j} c_{j \uparrow}^{\dagger} c_{j \uparrow} c_{j \downarrow}^{\dagger} c_{j \downarrow}
$$

where the first and second terms describe electron hopping and on-site Coulomb interaction, respectively. For the nearest-neighbor hopping, $t_{i j}$ is taken to be $t$; for the second-nearestneighbor hopping, $t_{i j}$ is taken to be $t^{\prime}$, and zero otherwise. Until Section 6 , we only consider $t^{\prime}=0$ cases. The parameters are assumed to satisfy the condition $U \gg t$.

When holes are doped in the half-filled system, all the doped holes are assumed to become small lattice polarons whose stabilization energy is large enough to support local vibrational states. Then, the effective transfer integrals for small polaron hopping become $t_{i j}$ multiplied by Frank-Condon factors of vibrational wave functions. The hole hopping probability is significantly reduced by the Frank-Condon factors; actually, we consider the extreme case where the hopping probability is reduced to zero for simplicity. Then, the effect of the doping is taken into account by eliminating hole occupied sites from accessible sites.

Usually, when the total Hamiltonian $H$ is split into the zeroth order Hamiltonian and a perturbation, the Coulomb interaction term in eq. (2.1),

$$
H_{U}=U \sum_{j} c_{j \uparrow}^{\dagger} c_{j \uparrow} c_{j \downarrow}^{\dagger} c_{j \downarrow}
$$

is taken as the zeroth order Hamiltonian, and the entire hopping term as a perturbation. If 
this splitting is employed, eq. (2.1) is reduced to the Heisenberg model,

$$
H_{H}=\frac{4 t^{2}}{U} \sum_{\langle n, m\rangle}\left(\mathbf{S}(n) \cdot \mathbf{S}(m)-\frac{1}{4} \hat{n}_{n} \hat{n}_{m}\right),
$$

where $\mathbf{S}(m)$ and $\hat{n}_{m}$ are the spin and number operators at site $m$, respectively, and the sum is taken over nearest spin pairs. ${ }^{17)}$ In this way, one reaches the conclusion that low-energy excitations are only spin excitations, and current flow does not occur. However, this conclusion is reversed if a new zeroth order Hamiltonian that contains a part of the hopping term is used.

Let us define new annihilation operators $a_{j}$ and $b_{j}$ to include a part of the hopping term; they are related to the original as

$$
\left(\begin{array}{c}
a_{j} \\
b_{j}
\end{array}\right)=e^{i \frac{\chi_{j}}{2}}\left(\begin{array}{cc}
e^{i \frac{\xi_{j}}{2}} \cos \frac{\theta_{j}}{2} & e^{-i \frac{\xi_{j}}{2}} \sin \frac{\theta_{j}}{2} \\
-e^{i \frac{\xi_{j}}{2}} \sin \frac{\theta_{j}}{2} & e^{-i \frac{\xi_{j}}{2}} \cos \frac{\theta_{j}}{2}
\end{array}\right)\left(\begin{array}{c}
c_{j \uparrow} \\
c_{j \downarrow}
\end{array}\right) .
$$

The angles $\xi_{j}$ and $\theta_{j}$ are azimuth and polar angles of the spin direction given by

$$
\begin{aligned}
& S_{x}(j)=\frac{1}{2}\left\langle c_{j \uparrow}^{\dagger} c_{j \downarrow}+c_{j \downarrow}^{\dagger} c_{j \uparrow}\right\rangle=\Delta_{j} \cos \xi_{j} \sin \theta_{j}, \\
& S_{y}(j)=\frac{i}{2}\left\langle-c_{j \uparrow}^{\dagger} c_{j \downarrow}+c_{j \downarrow}^{\dagger} c_{j \uparrow}\right\rangle=\Delta_{j} \sin \xi_{j} \sin \theta_{j}, \\
& S_{z}(j)=\frac{1}{2}\left\langle c_{j \uparrow}^{\dagger} c_{j \uparrow}-c_{j \downarrow}^{\dagger} c_{j \downarrow}\right\rangle=\Delta_{j} \cos \theta_{j},
\end{aligned}
$$

where $\langle\hat{O}\rangle$ denotes the expectation value of the operator $\hat{O}$, and $\Delta_{j}$ is the magnitude of the local spin moment at the $j$ th site.

In the following, we take $\xi_{j}$ and $\theta_{j}$ as adiabatic parameters. This may be justifiable since spin fluctuation described by variations of $\xi_{j}$ and $\theta_{j}$ will cost energy in the order of $t^{2} / U$, and electron hopping energy is in the order of $t$; thus, the former motion may be treated as a much slower one than the latter.

Note also that a phase factor $e^{i \frac{\chi_{j}}{2}}$ is added in eq. (2.4) so that the transformation matrix is single-valued; without it, sign ambiguity occurs with respect to the phase shift $\xi_{j} \rightarrow \xi_{j}+2 \pi$, although $\xi_{j}$ and $\xi_{j}+2 \pi$ are physically equivalent. The added phase factor $e^{i \frac{\chi_{j}}{2}}$ compensates the sign change of $e^{ \pm i \frac{\xi_{j}}{2}}$ by its sign change $e^{i \frac{\chi_{j}+2 \pi}{2}}=-e^{i \frac{\chi_{j}}{2}}$. An example for $\chi$ is $\chi=\xi$ but many other choices are possible. A similar phase factor is known to appear in the context of the Berry phase effect. ${ }^{14-16)}$ As will be seen later, this phase $\chi$ is crucial for the current generation.

Using the new operators, the electron hopping term in eq. (2.1) becomes $K_{a}+K_{b}+K_{a b}$, where $K_{a}, K_{b}$, and $K_{a b}$ are, respectively, given by

$$
\begin{aligned}
K_{a}= & -\sum_{k, j} t_{k j} e^{\frac{i}{2}\left(\chi_{k}-\chi_{j}\right)}\left(e^{i \frac{\xi_{k}-\xi_{j}}{2}} \cos \frac{\theta_{k}}{2} \cos \frac{\theta_{j}}{2}\right. \\
& \left.+e^{-i \frac{\xi_{k}-\xi_{j}}{2}} \sin \frac{\theta_{k}}{2} \sin \frac{\theta_{j}}{2}\right) a_{k}^{\dagger} a_{j},
\end{aligned}
$$


J. Phys. Soc. Jpn.

$$
\begin{aligned}
K_{b}= & -\sum_{k, j} t_{k j} e^{\frac{i}{2}\left(\chi_{k}-\chi_{j}\right)}\left(e^{i \frac{\xi_{k}-\xi_{j}}{2}} \sin \frac{\theta_{k}}{2} \sin \frac{\theta_{j}}{2}\right. \\
& \left.+e^{-i \frac{\xi_{k}-\xi_{j}}{2}} \cos \frac{\theta_{k}}{2} \cos \frac{\theta_{j}}{2}\right) b_{k}^{\dagger} b_{j}, \\
K_{a b} & =\sum_{k, j} t_{k j} e^{\frac{i}{2}\left(\chi_{k}-\chi_{j}\right)}\left(e^{i \frac{\xi_{k}-\xi_{j}}{2}} \cos \frac{\theta_{k}}{2} \sin \frac{\theta_{j}}{2}\right. \\
& \left.-e^{-i \frac{\xi_{k}-\xi_{j}}{2}} \sin \frac{\theta_{k}}{2} \cos \frac{\theta_{j}}{2}\right) a_{k}^{\dagger} b_{j} \\
& +\sum_{k, j} t_{k j} e^{\frac{i}{2}\left(\chi_{k}-\chi_{j}\right)}\left(e^{i \frac{\xi_{k}-\xi_{j}}{2}} \sin \frac{\theta_{k}}{2} \cos \frac{\theta_{j}}{2}\right. \\
& \left.-e^{-i \frac{\xi_{k}-\xi_{j}}{2}} \cos \frac{\theta_{k}}{2} \sin \frac{\theta_{j}}{2}\right) b_{k}^{\dagger} a_{j} .
\end{aligned}
$$

Let us consider the following state vector:

$$
|0\rangle=\prod_{j} a_{j}^{\dagger}|\mathrm{vac}\rangle
$$

where $j$ runs over all the accessible sites. This state vector is an eigenvector for $K_{a}+K_{b}+H_{U}$ with the energy eigenvalue zero;

$$
\left(K_{a}+K_{b}+H_{U}\right)|0\rangle=0 .
$$

The $\Delta_{j}$ for $|0\rangle$ is $\frac{1}{2}$. We take $|0\rangle$ as the zeroth order ground state vector; we call the energy band for $K_{a}$ the lower band, and that for $K_{b}$ the upper band.

Actually, the state vector

$$
\left|0_{b}\right\rangle=\prod_{j} b_{j}^{\dagger}|\operatorname{vac}\rangle
$$

also satisfies

$$
\left(K_{a}+K_{b}+H_{U}\right)\left|0_{b}\right\rangle=0
$$

thus, it is another zeroth order ground state vector. This state corresponds to the case $\xi_{j}^{\prime}=$ $\xi_{j}+\pi, \theta_{j}^{\prime}=-\theta_{j}+\pi$; it is far away from $|0\rangle$ in the adiabatic parameter space; thus, it will be difficult to reach $\left|0_{b}\right\rangle$ from $|0\rangle$ by perturbations in a reasonable time scale. Therefore, we treat $|0\rangle$ as an isolated zeroth order ground state in the following.

Lastly, in this section we would like to mention the phase factor $e^{i \frac{\chi_{j}}{2}}$ included in eq. (2.4). As is seen in eqs. (2.6)-(2.8), it induces a vector potential given by

$$
\mathbf{A}_{\text {fic }}=-\frac{\hbar}{2 q} \nabla \chi
$$

in all the hopping terms $K_{a}, K_{b}$, and $K_{a b}$, where $q=-e$ is the electron charge. It is not a vector potential for a true magnetic field; thus, it is often called "the vector potential for a fictitious magnetic field".

Note that the phase factor $e^{i \frac{\xi_{j}}{2}}$ multiplied to $c_{j \uparrow}$, and $e^{-i \frac{\xi_{j}}{2}}$ multiplied to $c_{j \downarrow}$ in eq. (2.4) 
also induce fictitious magnetic fields. The former induces the vector potential for up-spin electrons and the latter induces that for down-spin electrons given, respectively, by

$$
\begin{aligned}
& \mathbf{A}_{\uparrow}=-\frac{\hbar}{2 q} \nabla \xi, \\
& \mathbf{A}_{\downarrow}=\frac{\hbar}{2 q} \nabla \xi .
\end{aligned}
$$

The fictitious magnetic fields produced by them are exactly opposite; thus, the current produced by the former is canceled by that produced by the latter. This point will be seen in Section 4 .

\section{Half-Filled Antiferromagnetism and Nagaoka Ferromagnetism}

Before showing that spin vortex excitations accompany loop currents, we derive the wellknown results in this section to demonstrate the usefulness of the new annihilation and creation operators. The lattice polaron effect is totally neglected in this section.

In the first example, we show that the ground state for a half-filled system exhibits an antiferromagnetic correlation. ${ }^{17)}$ The second-order energy by the perturbation $K_{a b}$ is calculated as

$$
\begin{aligned}
E_{0}^{(2)} & =\left\langle 0\left|K_{a b} \frac{1}{-K_{a}-K_{b}-H_{U}} K_{a b}\right| 0\right\rangle \\
& \approx\left\langle 0\left|K_{a b} \frac{1}{-H_{U}} K_{a b}\right| 0\right\rangle \\
& =-\frac{t^{2}}{U} \sum_{\langle n, m\rangle}\left[1-\mathbf{n}\left(\xi_{n}, \theta_{n}\right) \cdot \mathbf{n}\left(\xi_{m}, \theta_{m}\right)\right],
\end{aligned}
$$

where $\mathbf{n}\left(\xi_{m}, \theta_{m}\right)$ is the unit vector with azimuth angle $\xi_{m}$ and polar angle $\theta_{m}$; the sum is taken over the nearest-neighbor pairs $\langle n, m\rangle$.

From eq. (3.16), it is apparent that the optimized spin direction is given by the condition

$$
\mathbf{n}\left(\xi_{n}, \theta_{n}\right) \cdot \mathbf{n}\left(\xi_{m}, \theta_{m}\right)=-1,
$$

which means that the optimal spin arrangement is antiferromagnetic. If the spin direction of a given site is fixed by a perturbation (say, $\xi_{0}$ and $\theta_{0}$ at the 0th site), all the other spin directions will be determined by optimization through the condition in eq. (3.17). Once this optimized arrangement is achieved, the system will remain there in an average sense for an experimental scale of time; then, an antiferromagnetic spin arrangement will be observed. It is worth noting that such an antiferromagnetic order is observed in the undoped cuprates. ${ }^{24)}$

In the second example, we examine the Nagaoka ferromagnetism that occurs in the system where a single hole is doped into the half-filled one. ${ }^{18)}$ In this case, an electron is removed from $|0\rangle$; thus, the lowest energy state is created by removing an electron from the highest single-particle energy state. 
Let us rewrite $K_{a}$ by using operators for extended "Bloch" states as

$$
K_{a}=\sum_{\alpha} \epsilon_{\alpha} \tilde{a}_{\alpha}^{\dagger} \tilde{a}_{\alpha}
$$

where $\tilde{a}_{\alpha}$ is related to $a_{j}$ by a unitary matrix $\left\{U_{\alpha j}\right\}$ as

$$
\tilde{a}_{\alpha}=\sum_{j} U_{\alpha j} a_{j}
$$

Then, the half-filled state vector is rewritten as

$$
|0\rangle=\prod_{\alpha} \tilde{a}_{\alpha}^{\dagger}|\mathrm{vac}\rangle
$$

The ground state for a system with one hole added to the half-filling is given by

$$
|\bar{\beta}\rangle=\tilde{a}_{\beta}|0\rangle .
$$

Noting that $\sum_{\alpha=1}^{N_{s}} \epsilon_{\alpha}=0$ ( $N_{s}$ is the number of sites), the total energy of $|\bar{\beta}\rangle$ is $-\epsilon_{\beta}$. It is apparent from eq. (2.6) that the value of $\epsilon_{\beta}$ is maximized if $\xi$ at all sites and $\theta$ at all sites are respectively equal. According to eq. (2.5), this corresponds to a ferromagnetic spin configuration, and the optimized ground state energy is given by

$$
E_{\beta}=-4 t
$$

this is the result obtained by Nagaoka. ${ }^{18)}$

\section{Loop Currents in the Effectively Half-Filled States}

In this section, we show that spin vortex excitations accompany loop currents by employing the perturbation theory. The zeroth order Hamiltonian in this section is

$$
H_{0}=K_{b}+H_{U}
$$

The condition given in eq. (3.17) is equal to

$$
\xi_{i}-\xi_{j}=\pi
$$

and

$$
\theta_{i}+\theta_{j}=\pi
$$

We will consider the spin vortex formation that violates the condition in eq. (4.24) while retaining the condition in eq. (4.25).

Using the condition in eq. (4.25), $K_{b}$ becomes

$$
K_{b}=-\sum_{k, j} t_{k j} e^{\frac{i}{2}\left(\chi_{k}-\chi_{j}\right)} \cos \frac{\xi_{k}-\xi_{j}}{2} \sin \theta_{k} b_{k}^{\dagger} b_{j} .
$$

This term is largest if $\theta_{k}$ is $\pi / 2$. We adopt this value so that a larger part of $H$ is included in $H_{0}$. In the following we consider a two-dimensional system. Taking the $z$-axis normal to the two-dimensional plane of the system, this leads to the confinement of the spin polarization in 
the two-dimensional plane. Then, the hopping terms are now written as

$$
\begin{aligned}
K_{a} & =-\sum_{k, j} t_{k j} e^{\frac{i}{2}\left(\chi_{k}-\chi_{j}\right)} \cos \frac{\xi_{k}-\xi_{j}}{2} a_{k}^{\dagger} a_{j}, \\
K_{b} & =-\sum_{k, j} t_{k j} e^{\frac{i}{2}\left(\chi_{k}-\chi_{j}\right)} \cos \frac{\xi_{k}-\xi_{j}}{2} b_{k}^{\dagger} b_{j}, \\
K_{a b} & =-i \sum_{k, j} t_{k j} e^{\frac{i}{2}\left(\chi_{k}-\chi_{j}\right)} \sin \frac{\xi_{k}-\xi_{j}}{2}\left(a_{k}^{\dagger} b_{j}+b_{k}^{\dagger} a_{j}\right) .
\end{aligned}
$$

We will use the above hopping terms from now on.

Before the perturbation calculation, we define the following approximate excited eigenvectors of $H_{0}$ :

$$
|\bar{i}, \alpha\rangle=\tilde{b}_{\alpha}^{\dagger} a_{i}|0\rangle,
$$

where $\tilde{b}_{\alpha}^{\dagger}$ is a creation operator for the extended "Bloch" state given by

$$
\tilde{b}_{\alpha}=\sum_{j} U_{\alpha j} b_{j}
$$

using operators for Bloch states, $K_{b}$ is diagonalized as

$$
K_{b}=\sum_{\alpha} \epsilon_{\alpha} \tilde{b}_{\alpha}^{\dagger} \tilde{b}_{\alpha}
$$

The eigenvalue for $|\bar{i}, \alpha\rangle$ is calculated as

$$
\begin{aligned}
H_{0}|\bar{i}, \alpha\rangle & =\epsilon_{\alpha} \tilde{b}_{\alpha}^{\dagger} a_{i}|0\rangle+U \sum_{j \neq i, \alpha} U_{\alpha j}^{*} b_{j}^{\dagger} a_{i}|0\rangle \\
& \approx\left(\epsilon_{\alpha}+U\right)|\bar{i}, \alpha\rangle
\end{aligned}
$$

where $U U_{\alpha i}^{*} b_{i}^{\dagger} a_{i}|0\rangle$ is added in going to the last line in eq. (4.33) by assuming that its addition is negligible.

Let us denote the ground state vector within the first-order perturbation in $H_{1}$ by

$$
\left|0^{\prime}\right\rangle=|0\rangle+|1\rangle,
$$

where the first-order correction $|1\rangle$ is calculated as

$$
|1\rangle=\sum_{m, j, \alpha} \frac{i t_{m j}|\bar{j}, \alpha\rangle}{U+\epsilon_{\alpha}} e^{\frac{i}{2}\left(\chi_{m}-\chi_{j}\right)} \sin \frac{\xi_{m}-\xi_{j}}{2} U_{\alpha m} .
$$

The operator for the $x$-component of current density with spin $\sigma$ is given by

$$
\hat{j}_{x \sigma}(l)=i t\left(c_{l+x \sigma}^{\dagger} c_{l \sigma}-c_{l \sigma}^{\dagger} c_{l+x \sigma}\right)
$$

where $l+x$ denotes the nearest-neighbor site of $l$ in the $x$ direction. ${ }^{25)}$ Note that it must be multiplied by $q=-e$ to obtain the electric current density, where $-e$ is the electron charge.

The expectation value for the up-spin current density is calculated as

$$
\left\langle 0^{\prime}\left|\hat{j}_{x \uparrow}(l)\right| 0^{\prime}\right\rangle
$$


J. Phys. Soc. Jpn.

$$
\begin{aligned}
& =t \sum_{m, \alpha} \frac{t_{m l} \sin \frac{\xi_{m}-\xi_{l}}{2} e^{\frac{i}{2}\left(\xi_{l}-\xi_{l+x}+\chi_{m}-\chi_{l+x}\right)} U_{l+x \alpha}^{-1} U_{\alpha m}}{2\left(U+\epsilon_{\alpha}\right)} \\
& -\quad t \sum_{m, \alpha} \frac{t_{m l+x} \sin \frac{\xi_{m}-\xi_{l+x}}{2} e^{\frac{i}{2}\left(\xi_{l+x}-\xi_{l}+\chi_{m}-\chi_{l}\right)} U_{l \alpha}^{-1} U_{\alpha m}}{2\left(U+\epsilon_{\alpha}\right)} \\
& +\quad \text { c.c. }
\end{aligned}
$$

and that for the down-spin current density is obtained as

$$
\begin{aligned}
& \left\langle 0^{\prime}\left|\hat{j}_{x \downarrow}(l)\right| 0^{\prime}\right\rangle \\
= & t \sum_{m, \alpha} \frac{t_{m l+x} \sin \frac{\xi_{m}-\xi_{l+x}}{2} e^{\frac{i}{2}\left(\xi_{l+x}-\xi_{l}-\chi_{m}+\chi_{l}\right)} U_{m \alpha}^{-1} U_{\alpha l}}{2\left(U+\epsilon_{\alpha}\right)} \\
- & t \sum_{m, \alpha} \frac{t_{m l} \sin \frac{\xi_{m}-\xi_{l}}{2} e^{\frac{i}{2}\left(\xi_{l}-\xi_{l+x}-\chi_{m}+\chi_{l+x}\right)} U_{m \alpha}^{-1} U_{\alpha l+x}}{2\left(U+\epsilon_{\alpha}\right)} \\
+ & \text { c.c. }
\end{aligned}
$$

If the dispersion in the upper band ( $\epsilon_{\alpha}$ in $K_{b}$ in eq. (4.32)) is neglected, eqs. (4.37) and (4.38) become

$$
\begin{aligned}
\left\langle 0^{\prime}\left|\hat{j}_{x \uparrow}(l)\right| 0^{\prime}\right\rangle & \approx \frac{t^{2}}{U} \sin \left(\xi_{l+x}-\xi_{l}\right), \\
\left\langle 0^{\prime}\left|\hat{j}_{x \downarrow}(l)\right| 0^{\prime}\right\rangle & \approx \frac{t^{2}}{U} \sin \left(\xi_{l}-\xi_{l+x}\right),
\end{aligned}
$$

where the identity $\sum_{\alpha} U_{l \alpha}^{-1} U_{\alpha m}=\delta_{l m}$ is used. According to eq. (4.39), the current in this case is considered to be generated by the fictitious magnetic fields given by the vector potentials $\mathbf{A}_{\uparrow}$ in eq. (2.14) for up-spin electrons, and $\mathbf{A}_{\downarrow}$ in eq. (2.15) for down-spin electrons. These fictitious magnetic fields are opposite, thus creating mutually opposite current densities. In the half-filled antiferromagnet, the nearest-neighbor spin pairs are antiparallel with $\xi_{j}-\xi_{k}=\pi$; therefore, both up- and down-spin current densities are zero.

According to eqs. (4.37) and (4.38), if $\nabla \chi$ is taken to be zero, the total current density is zero since the up- and down-spin current densities satisfy the relation

$$
\left\langle 0^{\prime}\left|\hat{j}_{x \downarrow}(l)\right| 0^{\prime}\right\rangle=-\left\langle 0^{\prime}\left|\hat{j}_{x \uparrow}(l)\right| 0^{\prime}\right\rangle, \text { if } \nabla \chi=0 ;
$$

the above is obtained using the fact that the unitary matrix $\left\{U_{\alpha m}\right\}$ can be made to be an orthogonal matrix (i.e., the relation $U_{m \alpha}^{-1}=U_{\alpha m}$ holds) if $\nabla \chi=0$. Therefore, for the appearance of the nonzero total current, nonzero $\nabla \chi$ is crucial; it can be said that the total current is produced by the fictitious magnetic field with vector potential $\mathbf{A}_{\text {fic }}$ in eq. (2.13).

The total energy for the state $\left|0^{\prime}\right\rangle$ is calculated as

$$
E=-\sum_{m, j, n, \alpha} \frac{t_{m j} t_{j n} \sin \frac{\xi_{m}-\xi_{j}}{2} \sin \frac{\xi_{n}-\xi_{j}}{2} U_{n \alpha}^{-1} U_{\alpha m} e^{\frac{i}{2}\left(\chi_{m}-\chi_{n}\right)}}{U+\epsilon_{\alpha}} .
$$


If the band dispersion is zero, eq. (4.41) becomes the energy calculated for the Heisenberg model using $|0\rangle$,

$$
E=-\frac{2 t^{2}}{U} \sum_{\langle n, m\rangle} \sin ^{2} \frac{\xi_{n}-\xi_{m}}{2}=\left\langle 0\left|H_{H}\right| 0\right\rangle,
$$

which is equivalent to the one obtained in eq. (3.16).

Let us now consider the energy formula in eq. (4.41) when a magnetic field $\mathbf{B}_{\mathrm{em}}=\nabla \times \mathbf{A}_{\mathrm{em}}$ ( $\mathbf{A}_{\mathrm{em}}$ is the electromagnetic vector potential) is present. The effect of the magnetic field is included by modifying the transfer integrals in the Hamiltonian as

$$
t_{m j} \rightarrow t_{m j} e^{-i \frac{q}{c} \int_{j}^{m} \mathbf{A}_{\mathrm{em}} \cdot d \mathbf{r}}
$$

by following the prescription by Peierls. Then, the unitary matrix $\left\{U_{\alpha m}\right\}$ and the energy dispersion become dependent on $\mathbf{A}_{\mathrm{em}}$ in general. However, we found that the following energy functional in which only the transfer integrals are modified according to eq. (4.43) in eq. (4.41) is useful;

$$
E\left[\mathbf{A}_{\mathrm{em}}\right]=-\sum_{m, j, n, \alpha} \frac{t_{m j} e^{-i \frac{q}{c} \int_{j}^{m} \mathbf{A}_{\mathrm{em}} \cdot d \mathbf{r}} t_{j n} e^{-i \frac{q}{c} \int_{n}^{j} \mathbf{A}_{\mathrm{em}} \cdot d \mathbf{r}} \sin \frac{\xi_{m}-\xi_{j}}{2} \sin \frac{\xi_{n}-\xi_{j}}{2} U_{n \alpha}^{-1} U_{\alpha m} e^{\frac{i}{2}\left(\chi_{m}-\chi_{n}\right)}}{U+\epsilon_{\alpha}}
$$

It can be easily checked that from the above functional, the $x$ component of current density is correctly obtained as

$$
j(l)_{x}=-\left.\frac{c}{q} \frac{\partial E\left[\mathbf{A}_{\mathrm{em}}\right]}{\partial A_{l+x l}}\right|_{\mathbf{A}_{\mathrm{em}}=0}=\left\langle 0^{\prime}\left|\hat{j}_{x \uparrow}(l)\right| 0^{\prime}\right\rangle+\left\langle 0^{\prime}\left|\hat{j}_{x \downarrow}(l)\right| 0^{\prime}\right\rangle
$$

where $A_{l+x l}$ is defined as

$$
A_{l+x l}=\int_{l}^{l+x} \mathbf{A}_{\mathrm{em}} \cdot d \mathbf{r} \approx \mathbf{A}_{\mathrm{em}}\left(\frac{\mathbf{r}_{l}+\mathbf{r}_{l+x}}{2}\right) \cdot \mathbf{e}_{x}
$$

with $\mathbf{e}_{x}$ being the unit vector in the $x$ direction and the lattice spacing is taken to be unity. The relation in eq. (4.45) indicates that $E\left[\mathbf{A}_{\mathrm{em}}\right]$ is the correct one up to the first order in $\mathbf{A}_{\mathrm{em}}$.

We further modify eq. (4.44) as

$$
E\left[\mathbf{A}_{\mathrm{em}}\right]=-\sum_{m, j, n, \alpha} \frac{t_{m j} t_{j n} \sin \frac{\xi_{m}-\xi_{j}}{2} \sin \frac{\xi_{n}-\xi_{j}}{2}\left|U_{n \alpha}^{-1} U_{\alpha m}\right| \cos \left(\frac{\chi_{m}-\chi_{n}}{2}+\beta_{m n}^{\alpha}-\frac{q}{c} A_{m n}\right)}{U+\epsilon_{\alpha}},
$$

where

$$
A_{m n}=\int_{n}^{m} \mathbf{A}_{\mathrm{em}} \cdot \mathbf{r}
$$

and $\beta_{m n}^{\alpha}$ is the phase of $U_{n \alpha}^{-1} U_{\alpha m}=\left|U_{n \alpha}^{-1} U_{\alpha m}\right| e^{i \beta_{m n}^{\alpha}}$.

We may use eq. (4.47) to calculate current when $\mathbf{A}_{\text {em }}$ is not zero, but very small; i.e., we assume that eq. (4.47) is a good one up to the second order in $\mathbf{A}_{\mathrm{em}}$. If we do so, we obtain

$$
j(l)_{x}\left[\mathbf{A}_{\mathrm{em}}\right]=\sum_{\substack{n ; \alpha ; \\\left(k, k^{\prime}\right)=(l, l+x) \\ \text { and }(l+x, l)}} \frac{2 t t_{n k^{\prime}} \sin \frac{\xi_{l+x}-\xi_{l}}{2} \sin \frac{\xi_{n}-\xi_{k^{\prime}}}{2}\left|U_{n \alpha}^{-1} U_{\alpha k}\right| \sin \left(\frac{\chi_{k}-\chi_{n}}{2}+\beta_{k n}^{\alpha}-\frac{q}{c} A_{k n}\right)}{U+\epsilon_{\alpha}}
$$


Note that $\left(\frac{\chi_{k}-\chi_{n}}{2}-\frac{q}{c} A_{k n}\right)$ is gauge invariant: under the gauge transformation

$$
\mathbf{A}_{\mathrm{em}}^{\prime}=\mathbf{A}_{\mathrm{em}}+\nabla f,
$$

single-particle Wannier wave functions, $\psi_{j}(\mathbf{x})$, are transformed as

$$
\psi_{j}^{\prime}(\mathbf{x})=\psi^{\prime}\left(\mathbf{x}-\mathbf{r}_{j}\right)=e^{-i \frac{q}{c} f(\mathbf{x})} \psi\left(\mathbf{x}-\mathbf{r}_{j}\right) \approx e^{-i \frac{q}{c} f\left(\mathbf{r}_{j}\right)} \psi_{j}(\mathbf{x}) ;
$$

therefore, hopping matrix elements transform as

$$
\begin{aligned}
\left\{t_{k j} e^{\frac{i}{2}\left(\chi_{k}-\chi_{j}\right)}\right\}^{\prime} & =e^{\frac{i}{2}\left(\chi_{k}-\chi_{j}\right)}\left\langle\psi_{k}^{\prime}|\hat{h}| \psi_{j}^{\prime}\right\rangle \\
& =e^{\frac{i}{2}\left(\chi_{k}-\chi_{j}\right)}\left\langle\psi_{k}|\hat{h}| \psi_{j}\right\rangle e^{i \frac{q}{c}\left(f_{k}-f_{j}\right)} \\
& =t_{k j} e^{\frac{i}{2}\left(\chi_{k}-\chi_{j}\right)+i \frac{q}{c}\left(f_{k}-f_{j}\right)},
\end{aligned}
$$

where $\hat{h}$ is the single-particle Hamiltonian; the above transformation indicates that the phase $\chi$ transforms as

$$
\chi^{\prime}=\chi+\frac{2 q}{c} f
$$

which makes the combination

$$
\frac{\chi_{k}-\chi_{n}}{2}-\frac{q}{c} A_{k n}
$$

gauge invariant.

Equation (4.49) can be further simplified if the gauge-invariant sum $\left(\frac{\chi_{k}-\chi_{n}}{2}-\frac{q}{c} A_{k n}\right)$ is small:

$$
j(l)_{x}\left[\mathbf{A}_{\mathrm{em}}\right] \approx \sum_{\begin{array}{c}
n ; \alpha ; \\
\left(k, k^{\prime}\right)=(l, l+x) \\
\text { and }(l+x, l)
\end{array}} \frac{2 t t_{n k^{\prime}} \sin \frac{\xi_{l+x}-\xi_{l}}{2} \sin \frac{\xi_{n}-\xi_{k^{\prime}}}{2}\left|U_{n \alpha}^{-1} U_{\alpha k}\right| \cos \beta_{k n}^{\alpha} \sin \left(\frac{\chi_{k}-\chi_{n}}{2}-\frac{q}{c} A_{k n}\right)}{U+\epsilon_{\alpha}} .
$$

The above expression is derived by assuming that eq. (4.47) is a good one up to the second order in $\mathbf{A}_{\mathrm{em}}$. This assumption will be supported in the mean-field calculation in Section 6, where we will obtain the current in accordance with the above expression (with $c^{-1} \mathbf{A}_{\mathrm{em}} \rightarrow 0$ ).

The current derived here most likely occurs as a collection of loop currents since the energy cost for the destruction of the antiferromagnetic spin arrangement is minimized by the collective creation of spin vortices with total winding number zero; ${ }^{26)}$ thus, the minimal energy spin vortex excitation is a creation of a nearby meron-antimeron pair ${ }^{26)}$ where "meron" is a spin vortex with winding number +1 , and "antimeron" is that with winding number -1 . In this way, only a small number of spin directions deviate from the antiferromagnetic arrangement, and the order of the excitation energy becomes $t^{2} / U$.

Spin vortices are characterized by integral topological numbers (winding numbers); thus, they are expected to be fairly stable. In addition, if the center of each spin vortex is located at a hole-occupied site, an additional stabilization will be obtained since it reduces the probability of the spin vortex destruction by the hopping motion of its center. It will also contribute to 
the reduction of the excitation energy since it increases the spin vortex core size and decreases the spin vortex core energy. In the rest of the work, we only consider cases where spin vortex centers are placed at hole-occupied sites.

\section{ARPES of Half-Filled States}

In the next section, we will examine some consequences of the presence of loop currents using a certain mean-field approximation to $H$. Before that, we show in this section that the ARPES of the half-filled state can be calculated by single Slater-determinant state vectors if a suitable choice of the mean-field is employed. We will use this mean-field approximation in the next section.

We first point out that the ground state given in eq. (2.9) can be written using another set of creation operators $\bar{a}_{\alpha}^{\dagger}$ as

$$
|0\rangle=\prod_{\alpha} \bar{a}_{\alpha}^{\dagger}|\mathrm{vac}\rangle
$$

where $\bar{a}_{\alpha}^{\dagger}$ is related to $a_{j}^{\dagger}$ by a unitary transformation

$$
\bar{a}_{\alpha}^{\dagger}=\sum_{j} C_{\alpha j} a_{j}^{\dagger}
$$

where $\left\{C_{\alpha j}\right\}$ is a unitary matrix.

We look for a particular choice for the unitary matrix $\left\{C_{\alpha j}\right\}$; in this choice, the ARPES intensity is calculated using matrix elements $\left\langle 0\left|\bar{a}_{\alpha}^{\dagger} c_{\mathbf{k} \sigma}\right| 0\right\rangle$. If such a unitary matrix is found, it will be possible to calculate the ARPES using a mean-field approximation.

Let us define a mean-field Hamiltonian for $H_{U}$ as

$$
\bar{H}_{U}=U \sum_{j}\left(\left\langle a_{j}^{\dagger} a_{j}\right\rangle b_{j}^{\dagger} b_{j}+\left\langle b_{j}^{\dagger} b_{j}\right\rangle a_{j}^{\dagger} a_{j}-\left\langle a_{j}^{\dagger} a_{j}\right\rangle\left\langle b_{j}^{\dagger} b_{j}\right\rangle\right),
$$

and try the mean-field Hamiltonian given by

$$
\bar{H}=K_{a}+K_{b}+K_{a b}+\bar{H}_{U}
$$

where $\left\langle a_{j}^{\dagger} a_{j}\right\rangle \approx 1$ and $\left\langle b_{j}^{\dagger} b_{j}\right\rangle \approx 0$ are assumed.

Taking $K_{a b}$ as a perturbation, the matrix elements for $\bar{H}$ with $\left\{a_{\ell}^{\dagger} \mid\right.$ vac $\left.\rangle\right\}$ as a basis are calculated as

$$
\begin{aligned}
& \left\langle\operatorname{vac}\left|a_{\ell}\left(K_{a}+K_{b}+\bar{H}_{U}\right) a_{\ell^{\prime}}^{\dagger}+a_{\ell} K_{a b} \frac{1}{-K_{a}-K_{b}-\bar{H}_{U}} K_{a b} a_{\ell^{\prime}}^{\dagger}\right| \mathrm{vac}\right\rangle \\
& =-t_{\ell \ell^{\prime}} e^{\frac{i}{2}\left(\chi_{\ell}-\chi_{\ell^{\prime}}\right)} \cos \frac{\xi_{\ell}-\xi_{\ell^{\prime}}}{2}-\sum_{k} \frac{t_{\ell k} t_{k \ell^{\prime}}}{U} e^{\frac{i}{2}\left(\chi_{\ell}-\chi_{\ell^{\prime}}\right)} \sin \frac{\xi_{\ell}-\xi_{k}}{2} \sin \frac{\xi_{k}-\xi_{\ell^{\prime}}}{2},
\end{aligned}
$$

which can be cast in an effective Hamiltonian given by

$$
H_{\text {eff }}=\sum_{\ell, \ell^{\prime}} t_{\ell \ell^{\prime}} e^{\frac{i}{2}\left(\chi_{\ell}-\chi_{\ell^{\prime}}\right)} \cos \frac{\xi_{\ell}-\xi_{\ell^{\prime}}}{2} a_{\ell^{\prime}} a_{\ell}^{\dagger}+\sum_{\ell, \ell^{\prime}, k} \frac{t_{\ell k} t_{k \ell^{\prime}}}{U} e^{\frac{i}{2}\left(\chi_{\ell}-\chi_{\ell^{\prime}}\right)} \sin \frac{\xi_{\ell}-\xi_{k}}{2} \sin \frac{\xi_{k}-\xi_{\ell^{\prime}}}{2} a_{\ell^{\prime}} a_{\ell}^{\dagger} .(5.61)
$$

This shows that if $\xi_{\ell}-\xi_{\ell^{\prime}} \approx \pi$, the first sum is small; the second term indicates that the band 
width is reduced to order $t^{2} / U$ from $t$ as is observed in the ARPES experiments. ${ }^{27)}$

We shall define the unitary matrix $\left\{C_{\alpha j}\right\}$ in eq. (5.57) as the one that diagonalizes $H_{\text {eff }}$ as

$$
H_{\mathrm{eff}}=\sum_{\alpha} \bar{\epsilon}_{\alpha} \bar{a}_{\alpha}^{\dagger} \bar{a}_{\alpha}
$$

Let us consider the expression for the ARPES intensity using the sudden approximation. In the sudden approximation with neglecting the matrix element effects, ${ }^{27)}$ the ARPES intensity for a momentum $\mathbf{k}$ is proportional to

$$
I(\mathbf{k}, \omega)=\sum_{s, \sigma}\left|\left\langle\Psi_{s}^{N-1}\left|c_{\mathbf{k} \sigma}\right| \Psi_{0}^{N}\right\rangle\right|^{2} \delta\left(\omega-E_{s}^{N-1}+E_{0}^{N}\right),
$$

where $\left|\Psi_{0}^{N}\right\rangle$ is a state with $N$ electrons and energy $E_{0}^{N}$, and $\left|\Psi_{s}^{N-1}\right\rangle$ indicates a state with $N-1$ electrons with energy $E_{s}^{N-1}$. For the case we are considering now, the former state and energy are given by $\left|\Psi_{0}^{N}\right\rangle=|0\rangle$ and $E_{0}^{N}=0$, respectively.

Now we express the $N-1$ electron state $\left|\Psi_{s}^{N-1}\right\rangle$ as

$$
\left|\Psi_{s}^{N-1}\right\rangle=\sum_{j} C_{s j}^{\prime}\left|\psi_{\ell}\right\rangle, \quad\left|\psi_{\ell}\right\rangle=a_{\ell}|0\rangle
$$

where we neglect the relaxation of a photo-created hole to a lattice polaron; namely, the small lattice polaron effect is neglected in $\left|\Psi_{s}^{N-1}\right\rangle$.

Taking $K_{a b}$ as a perturbation, the matrix elements for $H$ with $\left\{\left|\psi_{\ell}\right\rangle\right\}$ as a basis are calculated as

$$
\begin{aligned}
& \left\langle\psi_{\ell}\left|\left(K_{a}+K_{b}+H_{U}\right)+K_{a b} \frac{1}{-K_{a}-K_{b}-H_{U}} K_{a b}\right| \psi_{\ell^{\prime}}\right\rangle \\
& \approx\left\{\begin{array}{ll}
t_{\ell \ell^{\prime}} e^{\frac{i}{2}\left(\chi_{\ell}-\chi_{\ell^{\prime}}\right)} \cos \frac{\xi_{\ell-\xi_{\ell^{\prime}}}}{2}+\sum_{k} \frac{t_{\ell k} t_{k \ell^{\prime}}}{U} e^{\frac{i}{2}\left(\chi_{\ell}-\chi_{\ell^{\prime}}\right)} \sin \frac{\xi_{\ell}-\xi_{k}}{2} \sin \frac{\xi_{k}-\xi_{\ell^{\prime}}}{2} & \ell \neq \ell^{\prime} \\
-\sum_{j, k} \frac{t_{j k}^{2}}{U} \sin ^{2} \frac{\xi_{j}-\xi_{k}}{2} & \ell=\ell^{\prime}
\end{array} .\right.
\end{aligned}
$$

The above matrix elements indicate that the effective Hamiltonian for the system in which an electron is removed from the half-filled state is given by

$$
\begin{aligned}
& H_{\mathrm{eff}}^{\prime}=\sum_{\ell \neq \ell^{\prime}, k} t_{\ell \ell^{\prime}}{ }^{\frac{i}{2}\left(\chi_{\ell}-\chi_{\ell^{\prime}}\right)} \cos \frac{\xi_{\ell}-\xi_{\ell^{\prime}}}{2}\left|\psi_{\ell}\right\rangle\left\langle\psi_{\ell^{\prime}}\right| \\
& +\sum_{\ell \neq \ell^{\prime}, k} \frac{t_{\ell k} t_{k \ell^{\prime}}}{U} e^{\frac{i}{2}\left(\chi_{\ell}-\chi_{\ell^{\prime}}\right)} \sin \frac{\xi_{\ell}-\xi_{k}}{2} \sin \frac{\xi_{k}-\xi_{\ell^{\prime}}}{2}\left|\psi_{\ell}\right\rangle\left\langle\psi_{\ell^{\prime}}\right| \\
& -\sum_{\ell, j, k} \frac{t_{j k}^{2}}{U} \sin ^{2} \frac{\xi_{j}-\xi_{k}}{2}\left|\psi_{\ell}\right\rangle\left\langle\psi_{\ell}\right| .
\end{aligned}
$$

We define the unitary matrix $\left\{C_{s j}^{\prime}\right\}$ in eq. (5.64) as that diagonalizes $H_{\text {eff }}^{\prime}$ as

$$
H_{\mathrm{eff}}^{\prime}=\sum_{s} E_{s}^{N-1}\left|\Psi_{s}^{N-1}\right\rangle\left\langle\Psi_{s}^{N-1}\right|
$$

By comparing eqs. (5.61) and (5.66), it is seen that the following relation holds:

$$
C_{\alpha j}=C_{\alpha j}^{*} .
$$


Using the above relation, the matrix element $\left\langle\Psi_{s}^{N-1}\left|c_{\mathbf{k} \sigma}\right| \Psi_{0}^{N}\right\rangle$ is rewritten as

$$
\begin{aligned}
\left\langle\Psi_{s}^{N-1}\left|c_{\mathbf{k} \sigma}\right| \Psi_{0}^{N}\right\rangle & =\sum_{j} C_{\alpha j}^{*}\left\langle\psi_{j}\left|c_{\mathbf{k} \sigma}\right| 0\right\rangle \\
& =\sum_{j} C_{\alpha j}\left\langle 0\left|a_{j}^{\dagger} c_{\mathbf{k} \sigma}\right| 0\right\rangle \\
& =\left\langle 0\left|\bar{a}_{\alpha}^{\dagger} c_{\mathbf{k} \sigma}\right| 0\right\rangle .
\end{aligned}
$$

This shows that the mean-field approximation used above is the one we are looking for.

The energy difference $E_{s}^{N-1}-E_{0}^{N}$ is calculated as

$$
E_{s}^{N-1}-E_{0}^{N}=-\bar{\epsilon}_{s}+C,
$$

where

$$
C=-\sum_{j, k} \frac{t_{j k}^{2}}{U} \sin ^{2} \frac{\xi_{j}-\xi_{k}}{2} ;
$$

thus, the ARPES intensity is obtained as

$$
I(\mathbf{k}, \omega) \approx \sum_{s, \sigma}\left|\left\langle 0\left|\bar{a}_{s}^{\dagger} c_{\mathbf{k} \sigma}\right| 0\right\rangle\right|^{2} \delta\left(\omega+\bar{\epsilon}_{s}-C\right) .
$$

In the next section, we use the above mean-field approximation to gain qualitative insights into loop current states. There, it is applied to situations where the conditions assumed in this section are relaxed.

\section{Mean-Field Solutions}

In this section, we use the mean-field approximation introduced in the previous section. We consider the case with nonzero $t^{\prime}$ here; actually, we take $U=8 t$ and $t^{\prime}=-0.2 t$ that are believed to be appropriate for cuprate superconductors. We will see that the loop current is essentially proportional to $\nabla \chi$ in accordance with eq. (4.55) (the limit $c^{-1} \mathbf{A}_{\mathrm{em}} \rightarrow 0$ is taken), and a collection of loop currents create an extended current region; the calculated ARPES and elastic neutron scattering cross section profiles are shown to exhibit salient features observed in cuprates.

The system we consider is a two-dimensional square lattice with $16 \times 16$ sites. The number of electrons is $\left(256-N_{h}\right)$, where $N_{h}$ is the number of doped holes. The mean field Hamiltonian is given by

$$
\bar{H}=K_{a}+K_{b}+K_{a b}+U \sum_{j}\left(\left\langle a_{j}^{\dagger} a_{j}\right\rangle b_{j}^{\dagger} b_{j}+\left\langle b_{j}^{\dagger} b_{j}\right\rangle a_{j}^{\dagger} a_{j}-\left\langle a_{j}^{\dagger} a_{j}\right\rangle\left\langle b_{j}^{\dagger} b_{j}\right\rangle\right) .
$$

We diagonalize it to

$$
\bar{H}=\sum_{s} \bar{\varepsilon}_{s} \bar{d}_{s}^{\dagger} \bar{d}_{s}-U \sum_{j}\left\langle a_{j}^{\dagger} a_{j}\right\rangle\left\langle b_{j}^{\dagger} b_{j}\right\rangle
$$

by self-consistently calculation, $\left\langle a_{j}^{\dagger} a_{j}\right\rangle$ and $\left\langle b_{j}^{\dagger} b_{j}\right\rangle$. 
$\xi_{j}$ is chosen as

$$
\xi_{j}=\pi\left(j_{x}+j_{y}\right)+\sum_{M} W(j, M)-\sum_{A} W(j, A),
$$

where the function $W(j, M)$ is given by

$$
W(j, M)=\tan ^{-1} \frac{j_{x}-M_{x}}{j_{y}-M_{y}}
$$

$j, M$, and $A$ indicate two-dimensional coordinates for the $j$ th site, the center of a meron (spin vortex with winding number +1 ), and the center of an antimeron (spin vortex with winding number -1$)$, respectively; they are expressed using their components as $j=\left(j_{x}, j_{y}\right)$, $M=\left(M_{x}, M_{y}\right)$, and $A=\left(A_{x}, A_{y}\right)$, respectively.

For the phase $\chi_{j}$, we choose the following:

$$
\chi_{j}=\xi_{j}-2 g_{j}
$$

where

$$
g_{j}=\sum_{M^{\prime}} W\left(j, M^{\prime}\right)-\sum_{A^{\prime}} W\left(j, A^{\prime}\right)
$$

Usually, a site with a meron center creates a counterclockwise loop current (winding number +1 loop current) due to the phase $W(j, M)$ in $\xi$ (see eq. (6.75)). If the same site is also included in the sum over $M^{\prime}$ in $\chi$, clockwise loop current (winding number -1 loop current) arises, instead, due to the phase $W(j, M)-2 W(j, M)=-W(j, M)$; namely, the term with sum over $M^{\prime}$ in eq. (6.78) reverses the current direction from what is expected from the $\chi=\xi$ choice. The term with sum over $A^{\prime}$ in eq. (6.78) does the same thing for the antimeron centers; i.e., it reverses the expected clockwise loop current to a counterclockwise loop current. Note that for a given spin configuration, the total wave function for the $\chi=\xi$ choice, $\Psi$, and that for the $\chi=\xi-2 g$ choice, $\Psi^{\prime}$, is related as

$$
\Psi^{\prime}=\exp \left(-i \sum_{k=1}^{N_{e}} g\left(\mathbf{x}_{k}\right)\right) \Psi .
$$

The two states $\Psi$ and $\Psi^{\prime}$ have the same energy since they are connected by "the gauge transformation".

The $x$ component of the current density is calculated using the current density operator given by

$$
\begin{aligned}
\hat{j}_{x}(l) & =i t e^{\frac{i}{2}\left(\chi_{l+x}-\chi_{l}\right)} \cos \frac{\xi_{l+x}-\xi_{l}}{2}\left(a_{l+x}^{\dagger} a_{l}+b_{l+x}^{\dagger} b_{l}\right) \\
& +t e^{\frac{i}{2}\left(\chi_{l+x}-\chi_{l}\right)} \sin \frac{\xi_{l+x}-\xi_{l}}{2}\left(a_{l+x}^{\dagger} b_{l}+b_{l+x}^{\dagger} a_{l}\right) \\
& +\frac{1}{\sqrt{2}} i t^{\prime} e^{\frac{i}{2}\left(\chi_{l+x+y}-\chi_{l}\right)} \cos \frac{\xi_{l+x+y}-\xi_{l}}{2} \\
& \times\left(a_{l+x+y}^{\dagger} a_{l}-a_{l}^{\dagger} a_{l+x+y}+b_{l+x+y}^{\dagger} b_{l}-b_{l}^{\dagger} b_{l+x+y}\right)
\end{aligned}
$$


J. Phys. Soc. Jpn.

$$
\begin{aligned}
& +\quad \frac{1}{\sqrt{2}} t^{\prime} e^{\frac{i}{2}\left(\chi_{l+x+y}-\chi_{l}\right)} \sin \frac{\xi_{l+x+y}-\xi_{l}}{2} \\
& \times \quad\left(a_{l+x+y}^{\dagger} b_{l}-a_{l}^{\dagger} b_{l+x+y}+b_{l+x+y}^{\dagger} a_{l}-b_{l}^{\dagger} a_{l+x+y}\right) \\
& +\quad \frac{1}{\sqrt{2}} i t^{\prime} e^{\frac{i}{2}\left(\chi_{l+x-y}-\chi_{l}\right)} \cos \frac{\xi_{l+x-y}-\xi_{l}}{2} \\
& \times \quad\left(a_{l+x-y}^{\dagger} a_{l}-a_{l}^{\dagger} a_{l+x-y}+b_{l+x-y}^{\dagger} b_{l}-b_{l}^{\dagger} b_{l+x-y}\right) \\
& +\quad \frac{1}{\sqrt{2}} t^{\prime} e^{\frac{i}{2}\left(\chi_{l+x-y}-\chi_{l}\right)} \sin \frac{\xi_{l+x-y}-\xi_{l}}{2} \\
& \times \quad\left(a_{l+x-y}^{\dagger} b_{l}-a_{l}^{\dagger} b_{l+x-y}+b_{l+x-y}^{\dagger} a_{l}-b_{l}^{\dagger} a_{l+x-y}\right) \\
& +\quad \text { h.c. }
\end{aligned}
$$

where $l+x$ denotes the nearest-neighbor site of $l$ in the $x$ direction; $l+x+y$ and $l+x-y$ denote the second-nearest-neighbor sites of $l$ in the $x+y$ and $x-y$ directions, respectively. In a similar manner, $\hat{j}_{y}(l)$ is defined and used to calculate the $y$ component of the current density (the electric current densities are obtained by the multiplication of the charge $q=-e$ on the current densities).

In Fig. 1, a case with $N_{h}=8$ is depicted. The spin configuration is shown in Fig. 1(a); there are four meron-antimeron pairs. The excitation energy from the zero-spin-vortex case is $0.31 t$, which is in the order of $t^{2} / U=0.125 t$ as is expected. The current density is depicted in Fig. 1(b), together with $\nabla \chi$. It is seen that the former is almost proportional to the latter in accordance with the current formula in eq. (4.55) in the limit $c^{-1} \mathbf{A}_{\mathrm{em}} \rightarrow 0$. In the present case, $c^{-1} \mathbf{A}_{\mathrm{em}}$ produced by the current is negligible; thus, this result will be taken as a support for the current formula in eq. (4.55). In Fig. 1(c), the function $D(\mathbf{x})$ that relates the current density and $\nabla \chi$ as

$$
|\mathbf{j}(\mathbf{x})|=D(\mathbf{x})|\nabla \chi(\mathbf{x})|
$$

is depicted. It is about 0.42 all over, except at and around hole occupied sites. In Fig. 1(d), the current density $\mathbf{j}$ and $D \nabla \chi$ are shown (note that they are multiplied by the factor 2); they are almost identical, indicating that the current is almost proportional to $\nabla \chi$. In addition, the similar calculation with $t^{\prime}=0$ yields the current density almost identical to that obtained with $t^{\prime}=-0.2 t$ (Fig. 2); therefore, it is suggested that the current density is insensitive to $t^{\prime}$, and only sensitive to $\nabla \chi$.

Let us now consider cases with the same spin texture (one in Fig. 1(a)) but with different current distributions; they are obtained by choosing different $\chi$ 's given in eq. (6.77). In Fig. 3, four different current distributions are depicted (in fact, the current density in Fig. 3(a) is the same as the one in Fig. 1(b)). Corresponding to four different $\mathbf{j}(\mathbf{x})$ 's, four different $D(\mathbf{x})$ 's are obtained; one for Fig. 1(b) is depicted in Fig. 1(c), and the other three for Figs. 3(c)-3(d) are depicted in Figs. 4(a)-4(c); they look very similar. In Fig. 4(d), the average of the four 


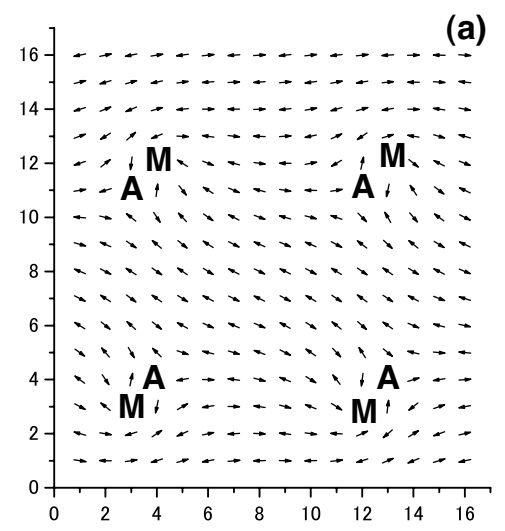

(a)

(b)

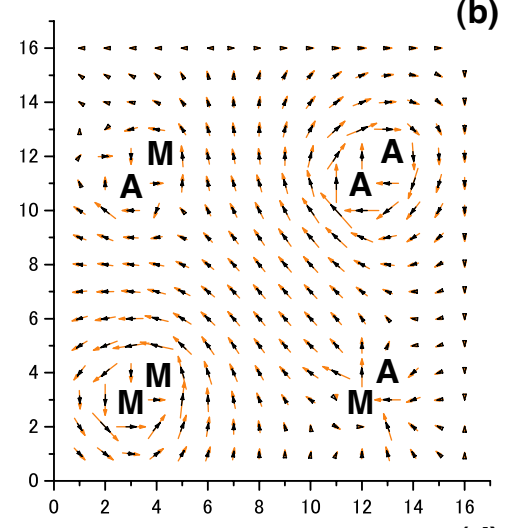

(c)
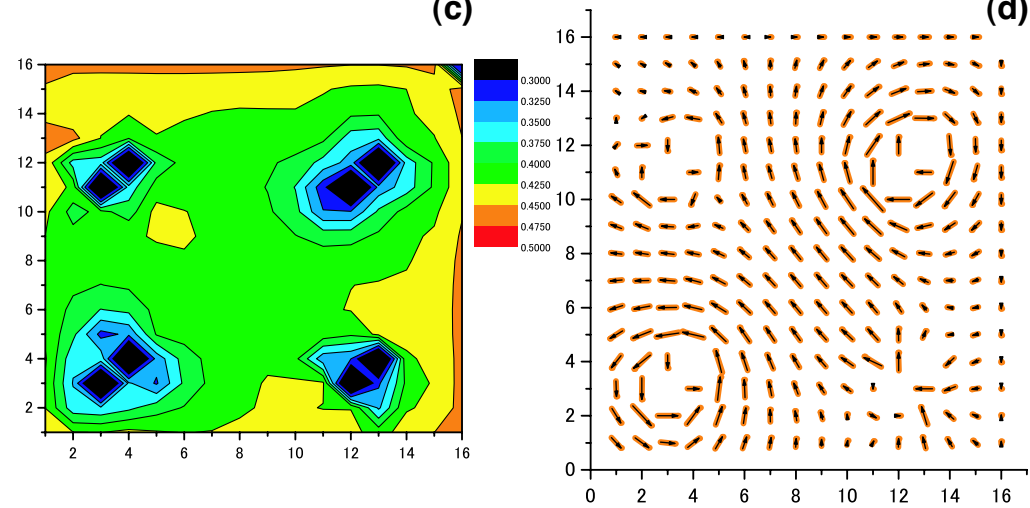

Fig. 1. (Color online) Plots obtained by mean-field calculations for an effectively half-filled system.

Calculations are performed for a two-dimensional $16 \times 16$ square lattice with open boundary conditions. Parameters used are $U=8 t$ and $t^{\prime}=-0.2 t$. The number of doped holes is 8 ; half of them are centers of merons and the rest are centers of antimerons. The total energy is $-13.17 t$, which is $0.31 t$ higher than the total energy for the zero-spin-vortex case. (a) Plot for spin configuration. Centers of spin vortices are indicated as "M" for a meron (winding number +1 spin vortex) and "A" for an antimeron (winding number -1 spin vortex), respectively. (b) Plot for current density $\mathbf{j}$ (short black arrows) and $\nabla \chi$ (long orange arrows). "M" and "A" here indicate centers of counterclockwise and clockwise loop currents, respectively; (c) Plot for $D(\mathbf{x})$, which connects $\mathbf{j}(\mathbf{x})$ and $\nabla \chi(\mathbf{x})$ as $|\mathbf{j}(\mathbf{x})|=D(\mathbf{x})|\nabla \chi(\mathbf{x})| ;$ (d) Plot for $2 \mathbf{j}$ (thick orange line; arrows are not attached but directions are the same as those of the black arrows) and $2 D(\mathbf{x}) \nabla \chi(\mathbf{x})$ (black arrows).

$D(\mathbf{x})$ 's, $D_{\text {aver }}(\mathbf{x})$, is depicted. Using $D_{\text {aver }}(\mathbf{x})$, an approximate current density is calculated as

$$
\mathbf{j}_{\text {ap }}=D_{\text {aver }}(\mathbf{x}) \nabla \chi(\mathbf{x})
$$

and depicted in Fig. 3. It is seen that $\mathbf{j}$ ap agrees very well with $\mathbf{j}$.

The above arguments suggest that we may obtain a good estimate of the electric current density using the average $D_{\text {aver }}(\mathbf{x})$ as

$$
\mathbf{j}_{e}(\mathbf{x}) \approx q D_{\text {aver }}(\mathbf{x})\left(\nabla \chi(\mathbf{x})-\frac{2 q}{c} \mathbf{A}_{\mathrm{em}}(\mathbf{x})\right)
$$

where a gauge potential term is added to make it gauge invariant. This is essentially the 


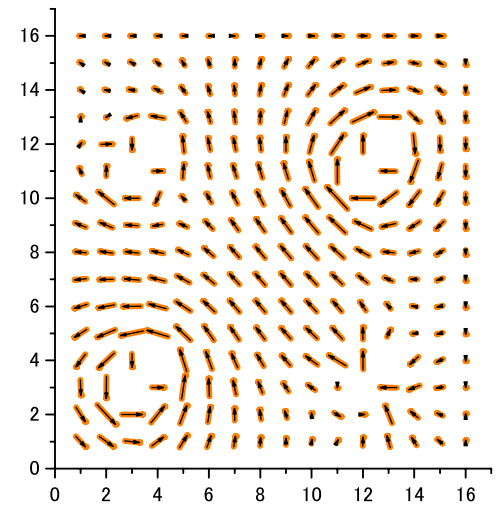

Fig. 2. (Color online) Plot of the current densities obtained by two different $t^{\prime}$ values. Other conditions are the same as in Fig. 1. The current density with $t^{\prime}=0$ is depicted using black arrows, while that with $t^{\prime}=-0.2 t$ is shown using thick orange lines (arrows are not attached, but directions are the same as those of the black arrows).

London equation for supercurrent; therefore, if $\nabla \chi$ is a stable physical variable, and eq. (6.83) is satisfied simultaneously with one of Maxwell's equations given by

$$
\frac{4 \pi}{c} \mathbf{j}_{e}(\mathbf{x})=\nabla \times\left(\nabla \times \mathbf{A}_{\mathrm{em}}(\mathbf{x})\right)
$$

the Meissner effect will be realized. Whether $\mathbf{j}_{e}(\mathbf{x})$ really gives rise to the Meissner effect is a very interesting subject; however, we defer its investigation as a future work.

We move on and calculate the ARPES and elastic neutron scattering profiles. The ARPES profile is calculated as

$$
\int_{\omega_{1}}^{\omega_{2}} d \omega \sum_{s, \sigma}\left|\left\langle 0\left|\bar{d}_{s}^{\dagger} c_{\mathbf{k} \sigma}\right| 0\right\rangle\right|^{2} \delta\left(\omega+\bar{\varepsilon}_{s}\right)
$$

where integration is performed from the energy of the highest occupied level to that of an arbitrary chosen lower level. In the following, we consider the case with 224 electrons (or 32 holes); then, $\omega_{2}$ is the energy for the 224 th level, and $\omega_{1}$ is taken to be that for the 210 th level.

The spin configuration $\mathbf{S}(j)$ is calculated using eq. (2.5); and the magnetic neutron elastic scattering cross section profile is calculated as

$$
\left|\sum_{j} e^{i \mathbf{k} \cdot \mathbf{x}_{j}} \mathbf{S}(j)\right|^{2}-k^{-2}\left|\sum_{j} e^{i \mathbf{k} \cdot \mathbf{x}_{j}} \mathbf{k} \cdot \mathbf{S}(j)\right|^{2} .
$$

In the following, we just report qualitative salient features of state with loop currents that may be relevant to the cuprate. In fact, there are many nearby energy states for given numbers of holes and spin vortices; however, quantitative comparison of the energy among those states will be dealt elsewhere. In Fig. 5, a case that exhibits a "Fermi arc"-like ARPES profile is displayed. ${ }^{19)}$ Two of the holes are centers of merons, another two are centers of antimerons, and the rest of the holes ( 28 holes) are simple vacancies. The phase $\chi$ is so chosen that all 
(a)

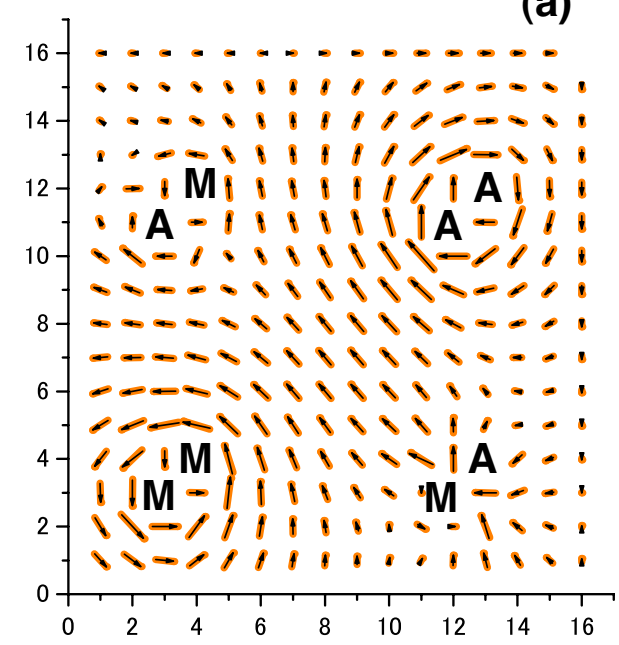

(c) (b)

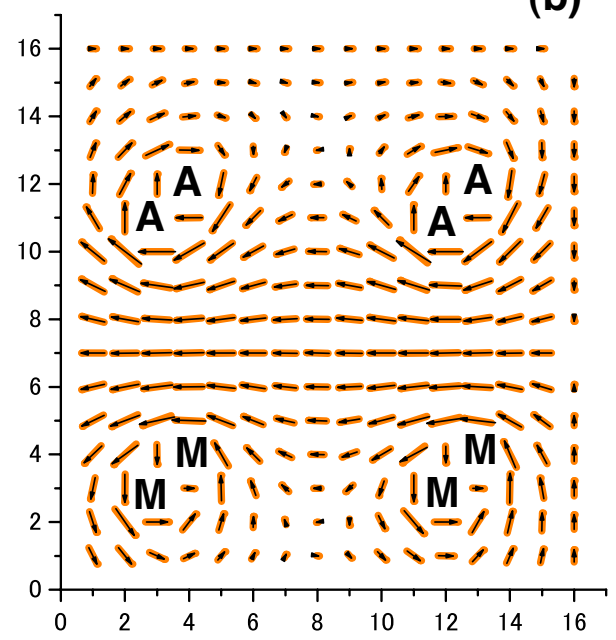

(d)
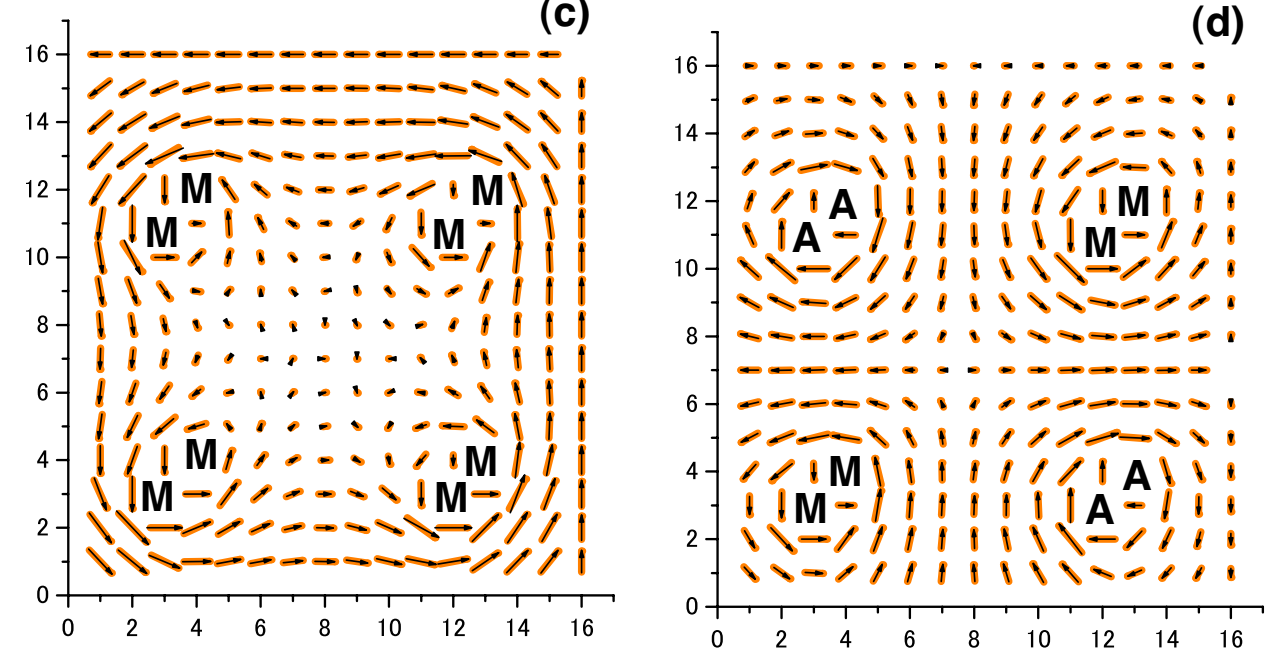

Fig. 3. (Color online) Plots of current densities $\mathbf{j}$ and approximate current densities $\mathbf{j}$ ap for four different $\nabla \chi$ 's. $2 \mathbf{j}$ is depicted by thick orange lines (arrows are not attached, but the directions are the same as those of $\mathbf{j}_{\mathrm{ap}}$ ), and $2 \mathbf{j}_{\mathrm{ap}}=2 D_{\text {aver }} \nabla \chi$ by black arrows.

four centers create counterclockwise loop currents. As a result, a collection of them creates an extended circular current region around them. In Fig. 5(b), a "Fermi arc" is seen. In this spin configuration, the neutron scattering cross section shows a single peak at $\left(k_{x}, k_{y}\right)=(\pi, \pi)$.

Although the current density distribution is insensitive to $t^{\prime}$, the ARPES profile is very sensitive to it. In Fig. 6, the ARPES profile calculated with $t^{\prime}=0$ is depicted, where the "Fermi arc" is lost.

If we change the current distribution as seen in Fig. 7(a) while keeping the same spin configuration, the "Fermi arc" also disappears. The current is now chosen as a collection of two counterclockwise loop currents plus two clockwise loop currents that creates a much smaller current flowing region. The comparison of Figs. 5 and 7 suggests that the Fermi arc is a sign of the presence of an extended current flowing region. 
(a)

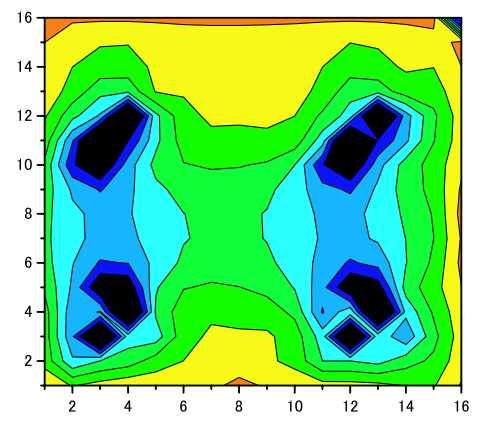

(c)

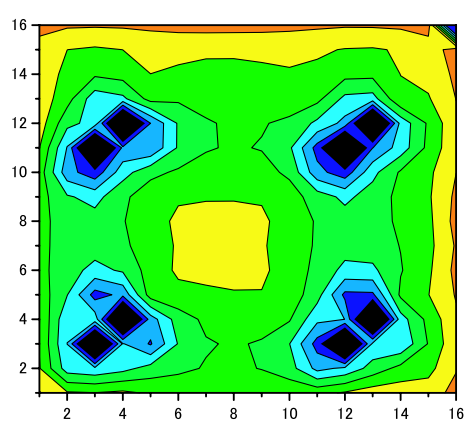

(b)

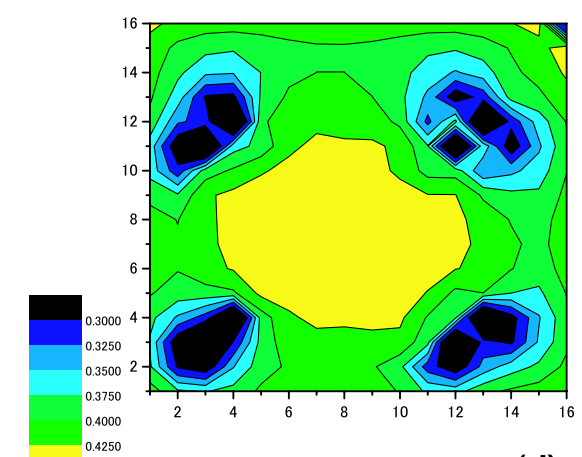

(d)

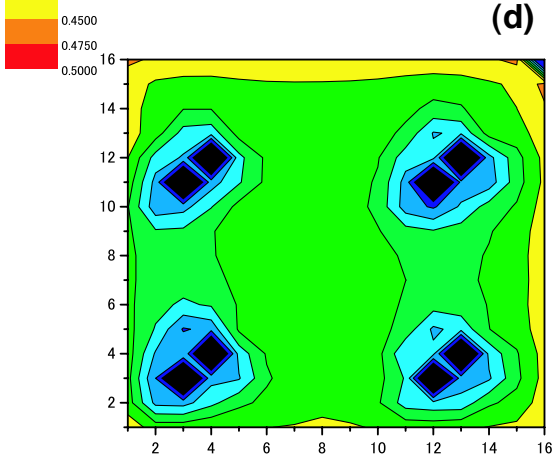

Fig. 4. (Color online) Plots for $D(\mathbf{x})$ obtained with the same condition as in Fig. 1, but with different $\nabla \chi$ choices. (a) $D(\mathbf{x})$, obtained with the $\nabla \chi$, that generates current in Fig. 3(b); (b) $D(\mathbf{x})$, obtained with the $\nabla \chi$, that generates current in Fig. $3(\mathrm{c}) ;(\mathrm{c}) D(\mathbf{x})$, obtained with the $\nabla \chi$, that generates current in Fig. 3(d). Plot for the average $D_{\text {aver }}(\mathbf{x})$ of the four $D(\mathbf{x})$ 's depicted in Fig. 1(c), Figs. 4(a)-4(c).

In Fig. 8, a case with a linear arrangement of holes is depicted. This hole configuration is chosen since it has been argued that stripes of doped holes are present in the cuprate. ${ }^{20-23,28)}$ The hole arrangement here corresponds to a stripe for the $x=0.125$ sample. Only 16 holes out of 32 are centers of spin vortices; 8 of them are meron centers and the others are antimeron centers. The total energy for this case is very slightly lower than the case in Fig. $5(0.04 t)$, which may be upset by the inclusion of the long-range Coulomb interaction or the lattice energy by the polaron interaction. The neutron scattering cross section profile exhibits a splitting of the peak, that is very similar to the one observed in cuprates. In fact, the splitting in the $k_{x}$ direction is exactly the one observed in the experiment with a $\frac{1}{8}$ shift from the $(1 / 2,1 / 2)$ point. $^{20-23)}$ The phase $\chi$ is so chosen that the centers for counterclockwise currents and those for clockwise currents line up in two separate lines. As is seen in Fig. 8(c), a river of current is created between the two lines. This figure suggests that a collection of loop currents will give rise to a macroscopic current. 
(a)

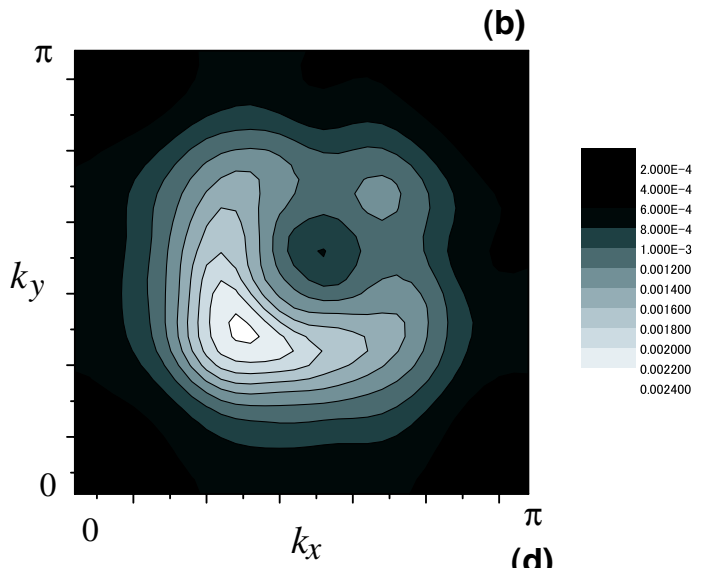

(c)
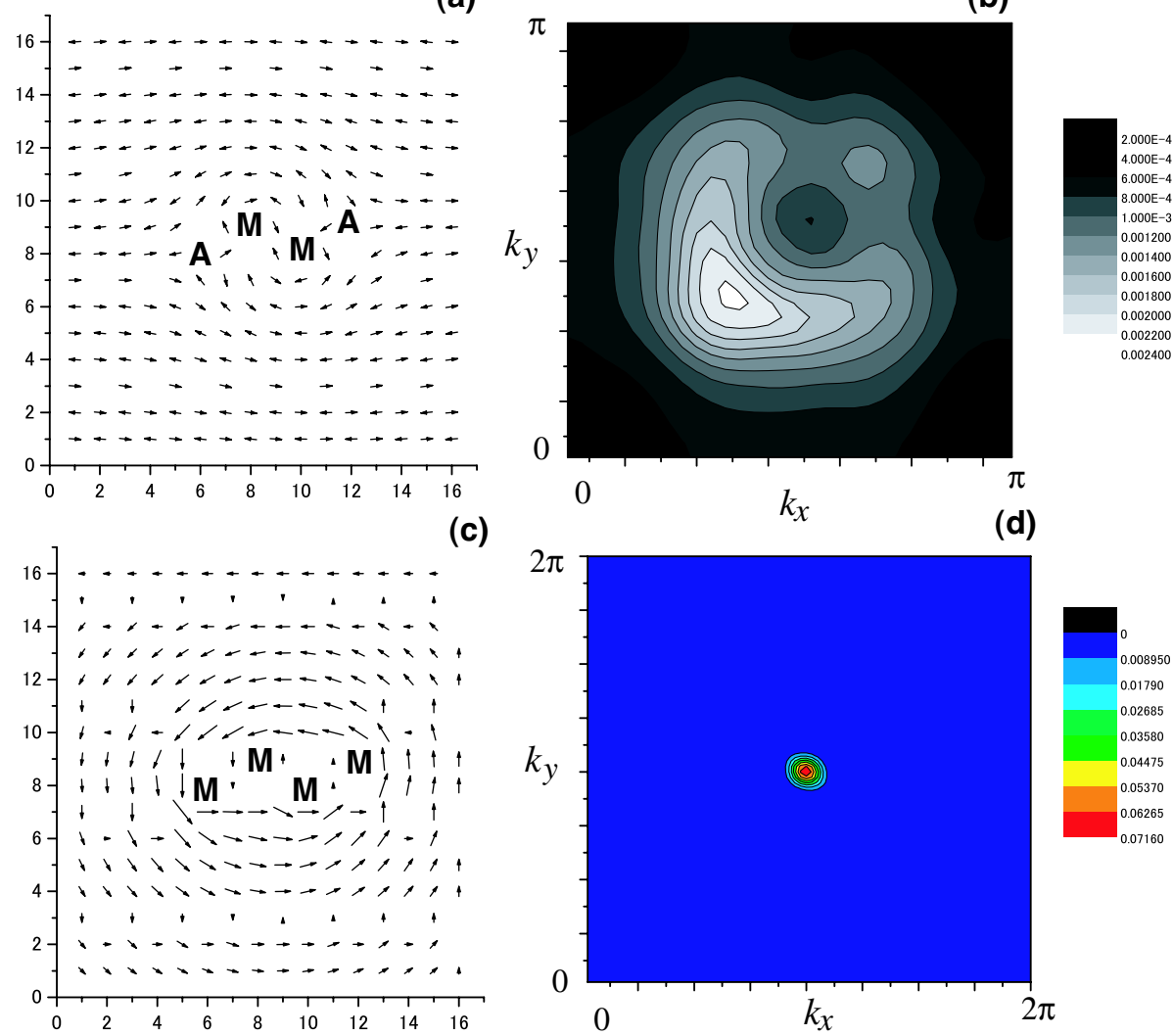

Fig. 5. (Color online) The same as Fig. 1, but with a different spin configuration, a different current distribution, and a different total number of holes, 32. The total energy is $-11.74 t$. (a) Plot for spin configuration. (b) Plot for current distribution. (c) Plot for ARPES profile in arbitrary units. $\omega_{2}=0.50 t$ and $\omega_{1}=0.29 t$. (d) Plot for neutron scattering cross section in arbitrary units.

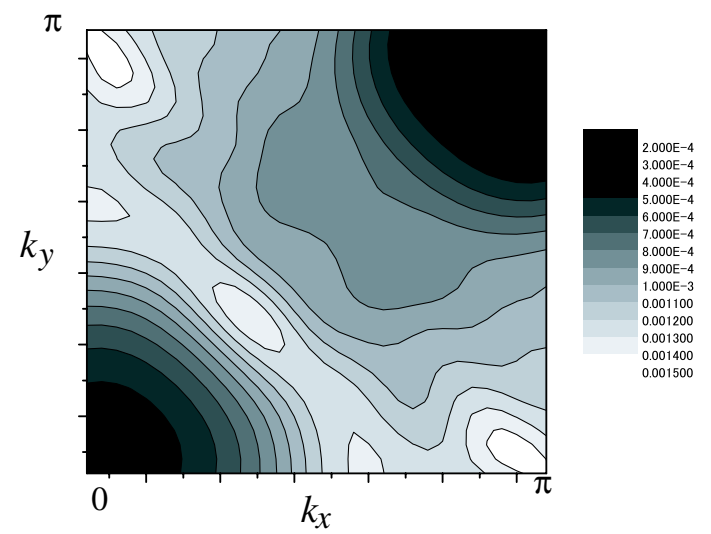

Fig. 6. Plot for ARPES profile. The conditions are the same as in Fig. 5, but with $t^{\prime}=0$. The total energy is $-11.53 t . \omega_{2}=1.17 t$ and $\omega_{1}=0.47 t$. 
(a)

(b)
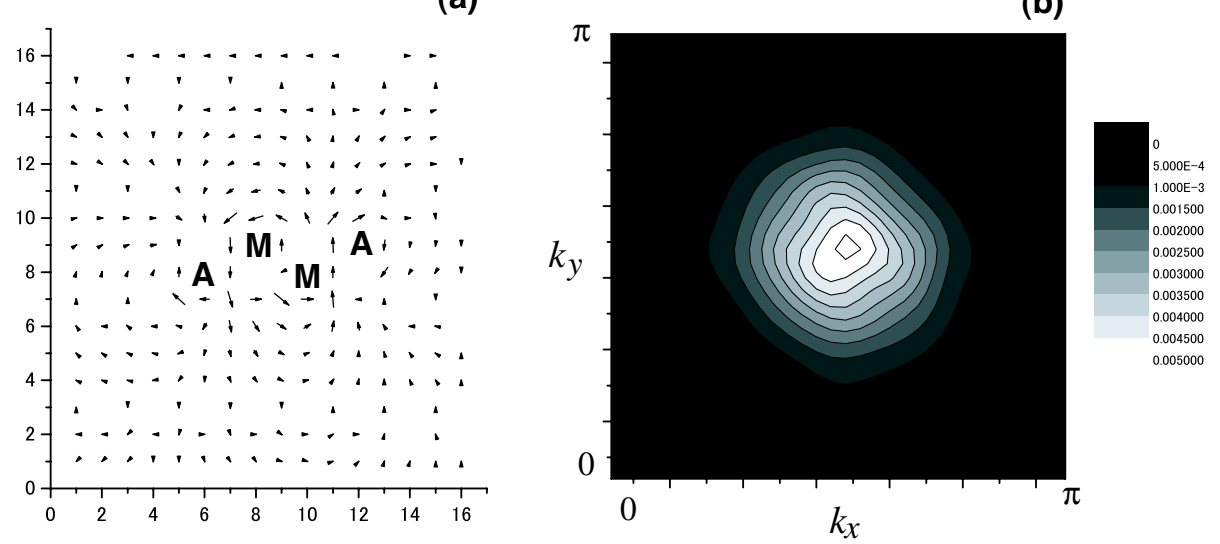

Fig. 7. The same as Fig. 5, but with a different current distribution. The total energy is $-11.74 t$. (a) Plot for current distribution. (b) Plot for ARPES profile. $\omega_{2}=0.50 t$ and $\omega_{1}=0.29 t$.

(a)

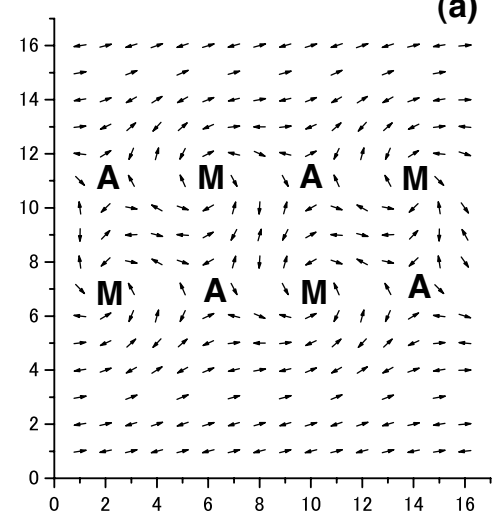

(c)

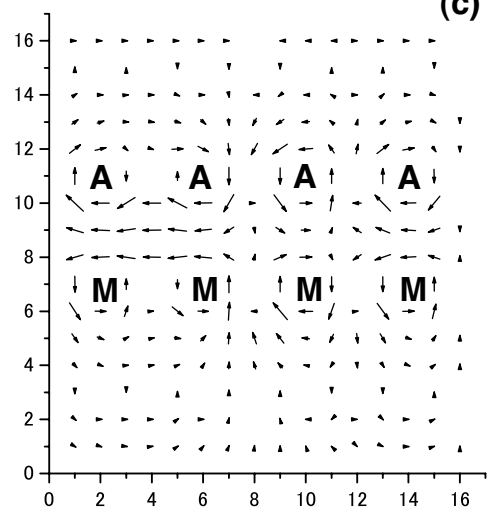

(b)

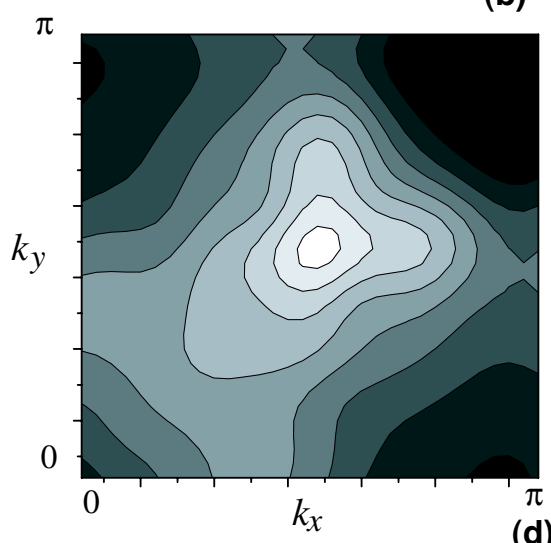

(d)

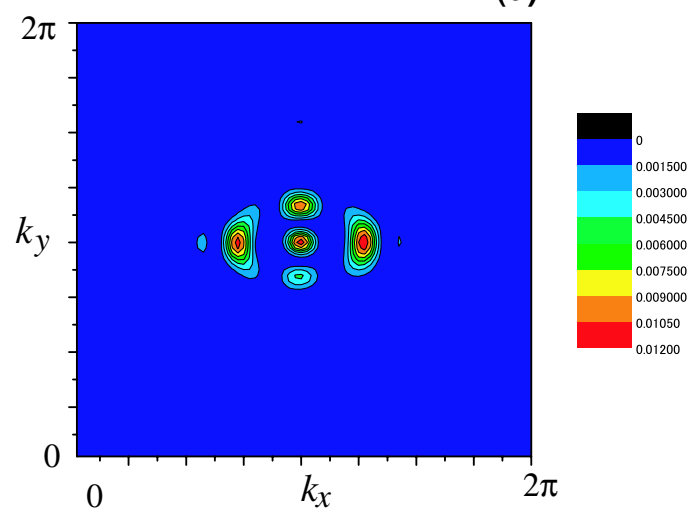

Fig. 8. (Color online) The same as Fig. 5, but with a different spin configuration and a different current distribution. The total energy is $-11.78 t$. (a) Plot for spin configuration. (b) Plot for current density. (c) Plot for ARPES profile. $\omega_{2}=0.94 t$ and $\omega_{1}=0.66 t$. (d) Plot for elastic neutron scattering cross section. 


\section{Concluding Remarks}

The present work shows that currents will flow around spin vortex centers due to the fictitious magnetic field that arises to ensure the single-valuedness of wave functions for electron hopping. The belief that low-energy excitations for the half-filled antiferromagnetic insulator are only those of spin degrees of freedom is reversed. A similar current generation mechanism may be possible in manganites if the spin degrees of freedom are replaced by the orbital degrees of freedom.

It is expected that spin vortices are so arranged that destruction of the antiferromagnetic order is localized to minimize the energy cost of their generation. Therefore, hopping matrix elements in $K_{b}$ are nonzero only in the vicinity of vortex centers; consequently, loop currents are rather localized. However, if the number of such loop currents is large and they are distributed over the sample, a macroscopic current may arise as an extension of the current flow seen in Fig. 8(c).

In the present treatment, fluctuations of $\xi$ and $\theta$ are neglected by assuming that they oscillate about mean values and only give rise to minor contributions. It is also assumed that the fluctuation of $\chi$ is negligible and the average value of $\chi$ is also intact. Large fluctuations that change the winding number of $\xi$ or $\chi$ are expected to be negligible at low temperatures. However, further work is necessary to clarify their stability against fluctuations. In reality, the doped carriers move more or less; the effect of the doped carrier hopping is also an important issue to be clarified.

By employing the mean-field approximation, ARPES profiles and elastic neutron scattering cross sections are calculated with parameters appropriate for cuprate superconductors. They exhibit some of the salient features observed in experiments. In particular, the calculated ARPES profiles suggest that the origin of the Fermi arc may be an extended loop current region, and the neutron scattering peak splitting may be due to the presence of a collinear order of spin vortices. In the cuprate, an extended current region with a collinear order of spin vortices may exist.

In the present work, we do not compare the energy for different spin configurations; there are many spin configurations with similar energies (for examples, ones seen in Figs. 5 and 8). To obtain realistic energies, however, the long-range Coulomb energy and the lattice energy for the polaron must be included. It is also important to include the magnetic field created by a collection of loop currents if the system is large since a current-carrying state should be higher in energy than a currentless state. The present work is a qualitative one; much more systematic and refined study is necessary to make quantitative comparison with experiments.

\section{Acknowledgment}

The author acknowledges helpful comments from S. Sugano and T. Yanagisawa. 


\section{References}

1) N. F. Mott: Proc. Phys. Soc. London A 62 (1949) 416.

2) M. Imada, A. Fujimori, and Y. Tokura: Rev. Mod. Phys. 70 (1998) 1039.

3) D. Mihailovic, C. M. Foster, K. Voss, and A. J. Heeger: Phys. Rev. B 42 (1990) 7989.

4) A. S. Alexandrov and N. Mott: Polarons and Bipolarons (World Scientific, Singapore, 1995).

5) A. Bianconi, N. L. Saini, A. Lanzara, M. Missori, T. Rossetti, H. Oyanagi, H. Yamaguchi, K. Oka, and T. Ito: Phys. Rev. Lett. 76 (1996) 3412.

6) G. Bersuker and J. B. Goodenough: Physica C 274 (1997) 267.

7) G. M. Zhao, V. Smolyaninova, W. Prellier, and H. Keller: Phys. Rev. Lett. 84 (2000) 6086.

8) O. Rösch, O. Gunnarsson, X. J. Zhou, T. Yoshida, T. Sasagawa, A. Fujimori, Z. Hussain, Z.-X. Shen, and S. Uchida: Phys. Rev. Lett. 95 (2005) 227002.

9) S. Miyaki, S. Uzuhara, K. Terada, Kenji Makoshi, and H. Koizumi: Phys. Rev. B 71 (2005) 85117.

10) L. Hozoi, S. Nishimoto, and A. Yamasaki, Phys. Rev. B 72 (2005) 144510.

11) H. Kamimura, H. Ushio, S. Matsuno, and T. Hamada: Theory of Copper Oxide Superconductors (Springer-Verlag, Berlin, 2005).

12) K. M. Shen, F. Ronning, W. Meevasana, D. H. Lu, N. J. C. Ingle, F. Baumberger, W. S. Lee, L. L. Miller, Y. Kohsaka, M. Azuma, M. Takano, H. Takagi, and Z.-X. Shen: Phys. Rev. B 75 (2007) 075115 .

13) S. Miyaki, K. Makoshi, and H. Koizumi: to appear in J. Phys. Soc. Japan.

14) M. V. Berry: Proc. Roy. Soc. London A 392 (1984) 45.

15) A. Shapere and F. Wilczek: Geometric Phases in Physics (World Scientific, Singapore, 1989).

16) A. Bohm, A. Mostfazadeh, H. Koizumi, Q. Niu, and J. Zwanziger: The Geometric Phases in Quantum Systems (Springer-Verlag, Berlin, 2003).

17) P. W. Anderson: Phys. Rev. 115 (1959) 2.

18) Y. Nagaoka: Phys. Rev. 147 (1966) 392.

19) M. R. Norman, H. Ding, N. Randeria, J. C. Campuzano, T. Yokoya, T. Takeuchi, T. Takahashi, T. Mochiku, K. Kadowaki, P. Guptasarma, and D. G. Hinks: Nature (London) 392 (1998) 157.

20) J. M. Tranquada, J. D. Axe, N. Ichikawa, Y. Nakamura, S. Uchida, and B. Nachumi: Phys. Rev. B 54 (1996) 7489.

21) H. Kimura, K. Hirota, H. Matsushita, K. Yamada, Y. Endoh, S.-H. Lee, C. F. Majkrzak, R. Erwin, G. Shirane, M. Greven, Y. S. Lee, M. A. Kastner, and R. J. Birgeneau: Phys. Rev. B 59 (1999) 6517.

$22)$ Y. S. Lee, R. J. Birgeneau, M. A. Kastner, Y. Endoh, S. Wakimoto, K. Yamada, R. W. Erwin, S.-H. Lee, and G. Shirane: Phys. Rev. B 60 (1999) 3643.

23) M. Fujita, K. Yamada, H. Hiraka, P. M. Gehring, S. H. Lee, S. Wakimoto, and G. Shirane: Phys. Rev. B 65 (2002) 064505.

24) D. Vaknin, S. K. Sinha, D. E. Moncton, D. C. Johnston, J. M. Newsam, C. R. safinya, and H. E. King, Jr.: Phys. Rev. Lett. 58 (1987) 2802.

25) D. J. Scalapino, S. R. White, and S. C. Zhang: Phys. Rev. Lett. 68 (1992) 2830.

26) M. Berciu and S. John: Phys. Rev. B 69 (2004) 224515.

27) A. Damascelli, Z. Hussain, and Z.-X. Shen: Rev. Mod. Phys. 75 (2003) 473.

28) S. A. Kivelson, I. P. Bindloss, E. Fradkin, V. Oganesyan, J. M. Tranquada, K. Kapitulnik, and C. 
J. Phys. Soc. Jpn.

Hawald: Rev. Mod. Phys. 75 (2003) 1201. 